\title{
Le film sur l'art Entre histoire de l'art et documentaire de création
}

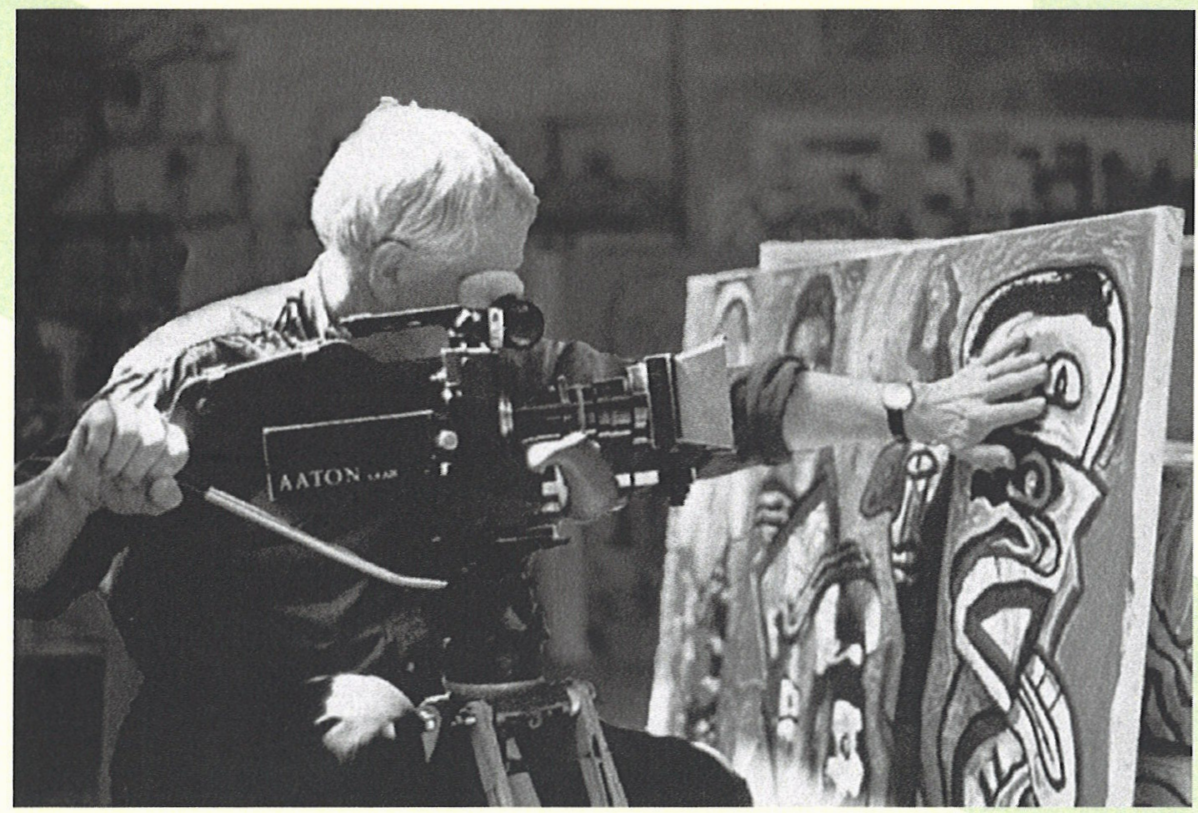

sous la direction de

Valentine Robert, Laurent Le Forestier et François Albera

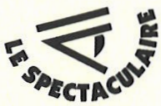


Sous la direction de

Valentine Robert, Laurenł Le Forestier, François Albera

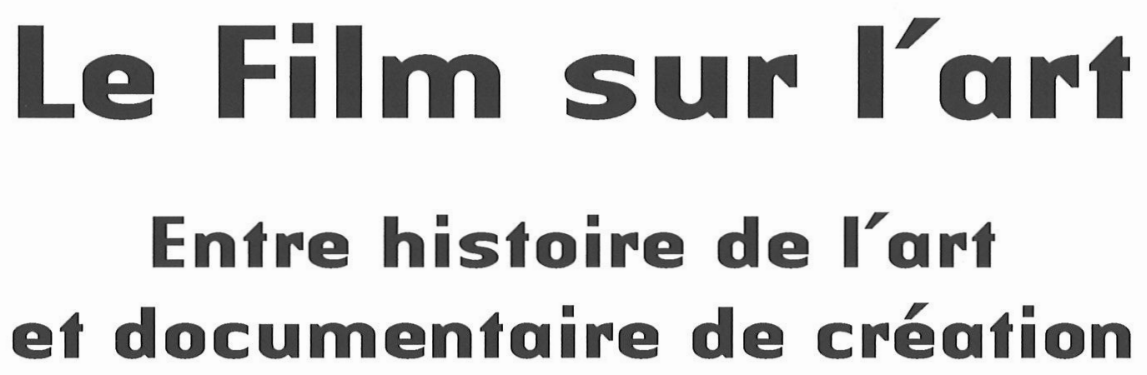

Avec la collaboration de

Kornelia Imesch et Mario Lüscher

Collection « Le Spectaculaire»

Presses universitaires de Rennes

2015 


\section{Remerciements}

Ce livre est issu d'un colloque international qui a pris place à l'université de Lausanne les 14, 15 et 16 avril 2011. Les directrices et directeurs du colloque François Albera, Kornelia Imesch, Laurent Le Forestier, Mario Lüscher et Valentine Robert tiennent à remercier toutes les personnes et institutions qui ont rendu possible l'organisation de cet événement scientifique et l'édition de cet ouvrage, plus particulièrement :

- l'équipe «Arts : pratiques et poétiques » de l'université Rennes 2

- la Cellule Recherche de l'UFR ALC de l'université Rennes 2

- le Centre des sciences historiques de la culture de l'université de Lausanne

- la Section d'histoire et esthétique du cinéma de l'université de Lausanne

- la Section d'histoire de l'art de l'université de Lausanne

- la faculté des lettres de l'université de Lausanne

- la Cinémathèque suisse

La rétrospective «Le film sur l'art. Approches d'un genre hybride " présentée à la Cinémathèque suisse en complément du colloque a été rendue possible par la collaboration du CNC (Centre national de la cinématographie), des AFF (Archives françaises du film), du musée du Louvre, et par la courtoisie des ayants droit. Nous tenons aussi à remercier personnellement Frédéric Maire, Chicca Bergonzi, Regina Bölsterli, Pascal Portner et Richard Szotyori de la Cinémathèque suisse.

La table ronde conjointe, intitulée "Conservation, promotion et diffusion du film sur l'art aujourd'hui ", a bénéficié du précieux concours de Philippe Kaenel, Gisèle Breteau-Skira, Antonie Bergmeier, Pascale Raynaud, Freddy Buache et Judith Wechsler, qui nous a également fait l'honneur, avec Hannes Brunner, d'une séance de présentation de leur travail de création de films sur l'art. 
Ces événements ont été filmés et sont disponibles sur le canal Youtube de la Cinémathèque suisse : "Colloque "Film sur l'art" - 15.04.2011" (www. youtube.com/watch? $v=Y 6 X c q \_J z p L M$, en 7 parties).

"Table ronde "Film sur l'art - 16.04.2011" (www.youtube.com/watch?v=iA74 JhdaADM, en 8 parties).

Nous remercions particulièrement Gilles Mouëllic, Laurence Bouvet-Lévêque; ainsi que Patricia Saugeon et Sylvain Portmann.

François Albera, Kornelia Imesch, Laurent Le Forestier et Mario Lüscher remercient tout particulièrement Valentine Robert pour son investissement dans l'organisation du colloque et l'édition de cet ouvrage. 


\section{F. Albera, L. Le Forestier, V. Robert, K. Imesch et M. Lüscher}

\section{Introduction}

\section{Approches d'un genre hybride, le film sur l'art}

Tous les visiteurs de musées sont accoutumés depuis de nombreuses années à faire halte (ou au contraire à contourner) les stations qu'on leur ménage devant des écrans où défilent entretiens avec les artistes, suites enchainées de leurs œuvres, commentaires explicatifs (biographiques, historiques) ou suppléments (contexte). Ces petits écrans succèdent aux salles où l'on projetait, il y a encore une vingtaine d'années, des montages de diapositives (généralement sur le modèle de la double projection et des fondus enchainés) ou des films sur pellicule.

C'est aussitôt après la Seconde Guerre mondiale que la réalisation de films sur l'art connut un spectaculaire développement : soutenues par diverses institutions culturelles, à commencer par l'Unesco, ces réalisations poursuivaient, certes, des expériences tentées avant-guerre ou pendant celle-ci ${ }^{1}$, qui avaient connu des réussites marquantes. En effet, l'importance prise par le cinéma éducatif dans les années 1930, conjuguée à la montée des nationalismes, explique pour une bonne part les prémices du genre qui visait souvent à mettre en valeur une culture nationale (voir par exemple Regards sur la Belgique ancienne de Storck, 1936). Mais le cinéma fut aussi, dès cette époque, utilisé à des fins d'enseignement des $\operatorname{arts}^{2}$ et

- 1 - En France un décret de 1941 rendant obligatoire la présence d'un documentaire dans les programmes des cinémas stimula la production et, selon Pierre Leprohon, donna naissance à " des genres à peu près ignorés autrefois : le documentaire artistique et le documentaire poétique " (L[eprohon] P., "Le documentaire est entré dans les mœurs ", Ciné-Mondial, nº 149, 21 juillet 1944, p. 6). En 1943 se tint le premier congrès du documentaire à Paris et l'un des deux conférenciers à intervenir était Sacha Guitry qui commenta Ceux de chez nous (1915).

- 2 - Voir Focillon H., "Note sur le cinématographe et l'enseignement des arts ", Bulletin de l'office international des instituts d'archéologie et d'histoire de l'art, vol. 1, n 1, 1934, p. 42-45. 
- 10. F. Albera, L. Le Forestier, V. Robert, K. Imesch er M. Lüscher

de conservation de la trace d'expositions rassemblant des toiles éparpillées dans le monde. C'est ainsi que René Huyghe tourna Rubens et son temps à l'occasion d'une grande exposition de l'ouvre du peintre à l'Orangerie.

Cependant ces films sur l'art de l'après-guerre manifestaient surtout l'émergence d'une «nouvelle alliance » entre des historiens de l'art, des conservateurs de musée, des critiques d'art ou des essayistes et des producteurs et des réalisateurs de cinéma qu'encadrait ou stimulait une demande institutionnelle. On parle volontiers d'un «âge d'or » du film sur l'art situé dans cette période. Il peut s'expliquer en partie par le renouveau de l'intérêt pour le genre documentaire (manifeste dans un événement comme le Congrès international du cinéma, à Bâle à la fin de l'été 1945), l'utopie très forte du cinéma comme vecteur idéal de connaissance (son rôle de média de communication et d'archivage), l'importance du circuit de distribution «non commercial » en Europe (ciné-clubs, filmguildes, etc.), les aides, dans divers pays européens, pour la production de courts métrages, la mobilisation en faveur du genre de certaines instances internationales (Unesco) ainsi que la constitution d'un organe international destiné à favoriser la création et la circulation de ces films (la Fédération internationale du film sur l'art) faisant fond sur cette situation et aidant à son développement.

De fait, cet « âge d'or " se poursuit durant quelques années (au moins jusque 1952, citée parfois comme année où s'amorce le déclin du genre) ${ }^{3}$, avant que la production ne s'affaiblisse, ou en tout cas paraisse perdre de l'intérêt du côté des médiateurs (journalistes, critiques, enseignants, exploitants de salles, distributeurs...) qui viennent à s'en agacer ${ }^{4}$. Elle continue toutefois une vie active mais souterraine, ayant ses manifestations propres, ses organisations (concurrentes), et trouve même parfois, occasionnellement, des débouchés : à l'occasion d'expositions et surtout dans le cadre de la télévision ${ }^{5}$. Mais encore faudrait-il faire le départ entre la production suscitée par ces deux types d'institutions productrices que sont les musées et les télévisions et l'accueil qu'elles font l'une et l'autre aux productions

- 3 - Si l'on s'en tient au cas de la production française de films sur l'art, elle angmente continument de 1946 à 1952, avant de décliner, notamment parce que trouvant un nouveau champ d'expansion à la télévision (ces statistiques sont données par BERTHOMÉ J.-P., « Les courts métrages d'art en France : 1946-1961 ", in Bü̈Her D. et Thomas F. [dir.], Le Court Métrage français de 1945 à 1968, de l'áge d'or aux contrebandiers, Rennes, Presses universitaires de Rennes, 2005, p. 95-109). L'année 1952 parait être également le pic de production de films sur l'art au niveau mondial, puisqu'on recense cette année-là environ 110 films produits.

- 4 - Voir Tallenay ].-L., "La peinture bavarde ou les Films sur l'Art ", Signes du temps, n 1 , janvier 1959.

- 5 - Voir à ce sujet Hamery R., "Filmer les arts aux débuts de la télévision : de la grande utopie éducative à la remise en question du média de masse comme outil pédagogique ", in Le Forestier L. et Mouëllic G. (dir.), Filmer l'artiste au travail, Rennes, Presses universitaires de Rennes, 2013, p. 277-288. 
élaborées en dehors d'elles, et pouvoir évaluer le type d'usages et d'approches que ces institutions réservent à ces productions-là, rarement appréhendées comme des œuvres appartenant à une conjoncture intellectuelle et cognitive mais plutôt comme une " ressource " anhistorique où puiser des extraits, des portraits, des gestes ou des paroles. Phénomène que les moyens de duplication et de diffusion actuels (supports numériques, Internet) ont, évidemment, démultiplié.

\section{Les relations art-cinéma}

Il serait vain de vouloir remonter le temps et fixer une origine historique aux noces de l'art, des arts, et du cinéma ${ }^{6}$ ! À la manière des caricaturistes du xix ${ }^{\mathrm{e}}$ siècle qui accompagnent l'émergence d'un public élargi - bientôt de masse - pour l'art, tant Georges Méliès, Émile Cohl que Prince-Rigadin, dès les premiers temps du cinéma, s'amusent ou brocardent la peinture ou la sculpture contemporaines : néo-impressionnisme, cubisme, abstraction... Le premier a collaboré à la presse (il est lui-même caricaturiste), le second également, issu du milieu des Hirsutes, il "invente " le monochrome à la façon d'Alphonse Allais. Plus fondamentalement le cinéma de Méliès, de Feuillade, de Griffith reprend la tradition des " tableaux vivants » qui fait florès sur la scène - et se poursuit jusqu'à Pasolini, Raul Ruiz ou Jean-Luc Godard chez qui il fait l'objet d'une réflexion touchant à la question de la représentation ${ }^{7}$. En excluant les films de fiction prenant une vie de peintre ou de sculpteur comme sujet ou requérant la collaboration d'un plasticien comme décorateur, on ne compte pas les peintres qui, dès les années 1910 et surtout au cours des années 1920, s'intéressent au film (Gromaire, Malevitch) et l'appréhendent comme nouveau support de leurs recherches plastiques (Richter, Léger, Man Ray, Dalí) ou comme ressource documentaire - comme ce fut le cas avec la photographie pour Delacroix, Courbet ou Munch. Ils trouveront une abondante postérité jusqu’à nos jours (où le cinéma est approprié par les artistes « multi-médias » sous diverses formes - Bruce Nauman, Matthew Barney, Pierre Huyghe, Philippe Parreno) après que nombre d'artistes eurent continué de

- 6 - Le binôme " peinture et cinéma » a tout de l' « auberge espagnole » : bon objet pour colloques, numéros spéciaux de revues, ouvrages, il amalgame des problèmes hétérogènes sans dessiner jamais une problématique. Depuis les premiers temps du cinéma on l'a mis en rapport avec Lascaux ou la tapisserie de Bayeux, on pressent le mouvement filmique chez certains peintres ou, à l'inverse, on reconnaît l'emprunt de l'art pictural au film dans telle composition d'image, tel usage de l'éclairage ou de la couleur. Ici le cinéma " relève " la peinture, là cette dernière ou ses récentes reformulations en art multimédia l'incorporent et le digèrent.

- 7 - Voir RoberT V., "La pose au cinéma : film et tableau en corps-à-corps ", Figures de l'art, $\mathrm{n}^{\circ} 23$ : Entre code et corps : tableau vivant et photographie mise en scène (BUIGNeT C. et RyKner A. [dir.]), printemps 2013, p. 73-89. 
pratiquer le film en parallèle de leur travail plastique (le groupe CoBrA, Yves Klein, Jacques Villeglé, Andy Wahrol, Bernhard Luginbühl, Richard Serra, Rebecca Horn...). Enfin le documentaire ou les actualités enregistrent des vues d'artistes, d'expositions en tant qu'événements culturels et contribuent par là à façonner l'image du " créateur » dans le public.

Filmer l'artiste au travail est plus rare et on accorde généralement au film de Sacha Guitry, Ceux de chez nous (1915), le statut de pionnier en la matière. Plusieurs fois ressorti dans des contextes fort différents et finalement montré par la télévision dans les années 1950, ce film conserve une capacité d'étonner le spectateur précisément parce qu'il ne s'inscrit pas dans la perspective explicative, didactique, démonstrative ou expressive qui sera celle des films sur l'art, mais demeure avant tout un document qu'on a pu qualifier avec quelque exagération de "brut " ou d' " authentique ${ }^{8}$ ». Il joue en tout cas un rôle-clé en 1944 dans le lancement du genre, au sein du documentaire, pendant l'Occupation de la France : on le projette en effet, commenté en direct par son auteur, lors du premier congrès du documentaire?. Dans les années 1950, la performance de Pablo Picasso enregistrée et mise en scène par Henri-Georges Clouzot aura également un impact de longue portée, générant dans la réflexion théorique comme dans l'approche même de la peinture, voire dans la pratique de celle-ci - via l'expérience tentée avec Jackson Pollock dans la même perspective -, un changement de régime.

\section{Le film sur l'art}

Le présent ouvrage procède d'un colloque international intitulé « Le film sur l'art : entre histoire de l'art et documentaire de création ", organisé par les universités de Rennes 2 et de Lausanne associées à la Cinémathèque suisse. Il a été conçu comme un " moment " au sein d'une recherche plus ample faisant l'objet d'un projet ANR, "Filmer la création", conduit par l'université Rennes 2, et qui a comporté d'autres volets, dans une perspective affirmée d'interdisciplinarité dont témoigne la collaboration des deux établissements et de plusieurs départements

- 8 - Comolli J.-L., "Le passé filmé ", Cahiers du Cinéma, n²77, juin 1977, p. 6-14.

- 9 - Ainsi Ceux de chez nous apparaît-il comme le documentaire par excellence, après une projection de " vieilles bandes, toujours jeunes, de Marey, Louis Lumière, Comandon, Alfred Chaumel et un extrait admirable de puissance et de virtuosité des Dieux du stade de Mme Léni Rieffenstahl (sic) ». (Ciné-Mondial, $\mathrm{n}^{\circ}$ 145, 23 juin 1944, p. 8) et s'inscrit-il dans le nouveau genre dont on cite -- dans un autre article un mois plus tard ("Le documentaire est entré dans les mœurs", art. cit.) un Rodin de René Lucot et un Lamartine de Jean Tedesco (au même moment l'Allemagne sort son Rembrandt de Hans Steinhoff). Indépendamment de ce mouvement sollicité par les autorités, Jean Lods avait tourné l'année précédente un Maillol, sculpteur qui est un portrait de l'artiste dont avant-guerre l'extrême-droite avait qualifié l'art de « boche ". 
(histoire de l'art, sciences historiques de la culture, histoire et esthétique du cinéma pour Lausanne et laboratoire de cinéma pour Rennes) ${ }^{10}$.

La rencontre lausannoise entendait s'attacher plus précisément à ce " genre " cinématographique hybride mais prolifique, le film sur l'art. Montrer l'artiste au travail (Matisse, Léger, Picasso, Masson, Pollock, Alechinsky, Molinier...), présenter la biographie d'un artiste disparu (le Michel Ange d'CErtel, 1940), sa vie intérieure (le Van Gogh de Resnais, 1948), son ouvre (Le Monde de Paul Delvaux, 1946 et le Rubens, 1948 de Storck et Haesaerts), entrer dans la logique de celle-ci pour en déplier la thématique ou les narrations qu'elle contient ( La Légende de Sainte Ursule d'Emmer, 1948), analyser sa construction formelle (les critofilms de Ragghianti), aborder une période ou suivre des évolutions stylistiques (De Renoir à Picasso, Haesaerts, 1950), autant de démarches qui associent, selon des modalités diverses, un cinéaste et un artiste ou un cinéaste et un historien de l'art (Gaston Diehl, Georg Schmidt, Jean Cassou, René Huyghe, James Johnson Sweeney...), quand l'historien de l'art ne se fait pas tout simplement cinéaste (Haesaerts, Ragghianti, Heilbronner). C'est que, de Warburg à Panofsky, de Focillon à Élie Faure et à Malraux, de Longhi à Francastel et à Damisch, il ne manque pas d'historiens de l'art, professionnels ou amateurs, qui se soient préoccupés - comme leurs devanciers le firent à propos de la photographie, de la diapositive (Grimm, Berenson, Wölfflin) - de ce que le cinéma pouvait apporter à leur discipline sur le plan de la simple documentation comme à celui du modèle de perception qu'induisent le découpage - les détails, les changements d'échelle -, le montage, la mise en mouvement enfin et la projection par transparence ${ }^{11}$.

Cantonné à de modestes tâches de pédagogie ou de diffusion culturelle, ou approché comme un nouvel outil heuristique, le film sur l'art a donc « triomphé » avant de connaître à la fois une généralisation - que la télévision décuple et banalise bien souvent (avec de brillantes exceptions dont les émissions Ways of Seeing dues à John Berger et ses collaborateurs en Grande-Bretagne) -, une conversion accrue à la fiction, soit mythologique soit démystificatrice (Van Gogh, Munch, Rembrandt, Basquiat...), et une qualification auteuriste avec des réussites exceptionnelles (ainsi le légendaire Mystère Picasso de Clouzot succédant à une tentative inachevée de Flaherty).

- 10 - Les actes d'un premier colloque reprenant le titre Filmer l'acte de création sont parus aux Presses universitaires de Rennes en 2009 (Frangne Pierre-Henry, Mouëllic Gilles, Viart Christophe, dir.). Un troisième centré sur "Les œuvres d'art dans le cinéma de fiction " (colloque tenu les 22, 23 et 24 mars 2012 sous la direction de Fiant Antony, Frangne Pierre-Henry et Mouëllic Gilles) complète ce triptyque (Rennes, PUR, coll. « Le Spectaculaire ", 2014).

- 11 - Voir Albera F., "Études cinématographiques et histoire de l'art », Perspective. La revue de I'INHA, $\mathrm{n}^{\circ}$ 3, 2006, p. 433-460. 
- 14 - F. Albera, L. Le Forestier, V. Robert, K. Imesch ei M. Lüscher

\section{Questions posées}

Le colloque avait pour première exigence d'envisager de manière documentée ce que fut la réalité du genre « film sur l'art » dans son histoire et dans son fonctionnement mais aussi dans ses doctrines et ses réalisations singulières, jusqu'aux dépassements contemporains où la démarche artistique croise la documentation.

Il entendait ensuite se demander si le film sur l'art avait contribué à la connaissance, avait inspiré, stimulé, bouleversé l'histoire de l'art. La présence d'historiens de l'art dans le mouvement d'après-guerre avec la création d'une Fédération internationale des films sur l'art (FIFA) a-t-elle laissé des traces? Y a-t-il désormais une histoire de l'art inséparable du nouveau médium?

Enfin il s'agissait de dégager les tendances de cette évolution du film sur l'art, des débats ayant eu lieu à ce sujet et de tirer un bilan de cette riche histoire devenue quelque peu opaque (où sont ces films? comment les voir?) jusqu'à la situation présente : où en sommes-nous aujourd'hui dans le domaine du film sur l'art? Qui le produit, quelle visée se donne-t-il, qui le voit et où?

Sur le plan des institutions qui ont ouvré à l'avènement du film sur l'art comme des systèmes d'aide qui en ont permis l'épanouissement au-delà des cénacles et festivals spécialisés, Laurent Le Forestier s'est ainsi employé à examiner l'entreprise d'après-guerre des "Amis de l'art » emmenée par Gaston Diehl et de l'association "Plaque tournante ", et François Albera à la mise en place et à l'évolution de la Fédération internationale des films sur l'art qui prend la suite de ces premières initiatives. Christel Taillibert a abordé la dimension politique, nationaliste de cette production sur le cas de l'Italie fasciste et Frédéric GimelloMesplomb la politique de la prime à la qualité qui entre en vigueur en 1954 en France et son impact sur le développement du « genre ». Au plan ensuite du projet même de recourir au film, dès l'avènement du Cinématographe Lumière, en histoire de l'art, Valentine Robert en a analysé les origines dans le cadre de l'enseignement de l'art par la projection d'images, postulant que l'écran avait eu un impact épistémologique majeur sur l'histoire de l'art. Puis différentes doctrines, mises en ouvre dans des séries de films, ont été abordées : le pionnier Paul Haesaerts dès les années 1930, dont l'entreprise de "cinéma critiquee " est étudiée par Céline Maes, son continuateur et contradicteur Carlo Ragghianti, dont Chiara Savettieri a analysé la théorie des « crito-films ", tous deux partisans d'une nouvelle méthode d'approche de l'œuvre d'art par le film. Antonio Costa détaille ces approches " critiques " en esquissant une typologie des films qui, entre le documentaire et l'essai, traitent du cas de l'art vénitien. La dimension idéologique qui sous-tend la représentation de l'art dans les actualités filmées a été saisie de manière générale par Mario Lüscher à partir du lien ménagé entre 
les « regardeurs » et le spectateur, et de manière particulière sur le cas suisse par Kornelia Imesch, avec la représentation des ouvres de Jean Tinguely dans le Ciné-Journal suisse, et sur les cas belge et français par Roxane Hamery, avec la représentation des arts africains dans les cinémas coloniaux entre 1945 et 1961. Les politiques de "programmation " de la télévision en matière de documentaires sur l'art ont fait l'objet des recherches d'Yves Chevrefils-Desbiolles sur le cas de Jean José Marchand et ses Archives du XX siècle, et de Martin Goutte sur celui de la chaîne franco-allemande Arte. L'objet que s'est donné Benoît Turquety avec le Cézanne de Straub et Huillet croise les problématiques institutionnelles et les démarches individuelles puisque le film s'est imposé en dehors des cadres qui l'avaient rendu possible, la commande du Musée d'Orsay qui le refusa. Enfin, outre celle-ci, quelques entreprises individuelles ont été examinées avec les enjeux chaque fois fort différents qu'elles comportent : Alain Boillat a analysé Ceux de chez nous de Sacha Guitry (1915-1952) que domine une conception discursive, orale et dont on a dit plus haut combien il occupe une place «fondatrice » dans la mise en place du genre, Fabienne Bonino a éclairé la production de films sur l'art d'Henri Storck, qui s'associait à des historiens d'art pour renouveler l'analyse des ouvres, Nicolas Brulhart a présenté la démarche de Gerry Schum, artiste conceptuel documentant par le film des interventions éphémères, enfin Judith Wechsler présente dans ce volume sa pratique d'historienne de l'art / réalisatrice de films sur l'art, faisant suite à une séance de projections d'extraits de ses films qui se tint durant le colloque à la Cinémathèque suisse. Pour présenter l'ensemble des études, notre volume a choisi un ordonnancement quadripartite s'attachant d'abord aux origines du film sur l'art (pédagogie et propagande), puis à l'institutionnalisation du genre (entre histoire de l'art et cinéma), le considérant ensuite, selon la formule bazinienne, comme une création au second degré, envisageant enfin ses déplacements du côté des actualités, de la télévision et du vidéo-art.

\section{Un billan historiographique}

Pour circonscrire la part que prennent les études du film sur l'art au sein de l'historiographie de l'art et du cinéma, et le désir que nous manifestons ici d'approfondir la recherche à cet endroit, il faut partir du fait que ces films se présentent la plupart du temps eux-mêmes comme des " essais historico-théoriques » de nature réflexive, qui " formulent ", appliquent ou vérifient des réflexions sur la relation entre le cinéma et les autres arts, et articulent une définition du genre lui-même (ses buts, ses fonctions, ses enjeux). Aussi devraient-ils être pris en compte non comme simples objets mais aussi sujets de ce bilan historiographique. Plusieurs 
- 16 - F. Albera, L. Le Foresiier, V. Robert, K. Imesch ei M. Lüscher

connaissent d'ailleurs des « extensions" d'ordre textuel, soit que certaines parties du film aient été retranscrites sur papier (comme les entretiens qui y prennent place, ou le commentaire), soit que des compléments (tels que des interviews, des recherches ou des documents supplémentaires) en accompagnent l'édition et ce pour mieux les faire exister sur le plan historiographique auquel ils ressortissent bel et bien. Essors bibliographique et filmographique seront donc, le plus souvent, étroitement imbriqués.

L'« avènement historiographique " du film sur l'art coïncide avec le moment d'émergence et d'institutionnalisation de cette production, après la Seconde Guerre mondiale. Cet avènement s'accompagne d'emblée d'une importante littérature, contemporaine d'une forte poussée théorique dans le champ du cinéma et d'un intérêt inédit (du moins dans les proportions qu’il atteint) des sciences humaines (philosophie, histoire de l'art, etc.) pour le cinéma. Parmi les textes importants qui voient le jour, il en est d'abord d'obédience institutionnelle, liés à la mise en place de la Fédération internationale du film sur l'art (FIFA), dès 1948. Ils visent à recenser, cataloguer et indexer, pour mieux diffuser et stimuler, cette production documentaire sur l'art, considérée comme le « ferment efficace d'un monde durablement pacifié ${ }^{12}$ ». Ces entreprises de catalogage international, soutenues par la FIFA comme par l'Unesco, ont pour résultats notoires et successifs (chaque édition complétant les listes précédentes) les publications suivantes ${ }^{13}$ : en 1949 , Le Film sur l'art: études critiques et catalogue international ${ }^{14}$; en 1951, Le Film sur l'art-Bilan 1950 : études critiques, répertoire international ${ }^{15}$; deux ans après, dirigé par Francis Bolen, Le Film sur l'art-Panorama 1953 : répertoire international illustré ${ }^{16}$, et la version romaine "détaillée » de cet inventaire, où les titres passent de 701 à 1109 (en provenance de 30 pays) et reçoivent tous un commentaire sous la direction de Carlo Ludovico Ragghianti : Le Film sur l'art : répertoire général international des films sur les arts ${ }^{17}$. On assiste

- 12 - Chevrefils Desbiolles Y., "Avant-propos ", Le film sur l'art et ses frontières, Aix-enProvence, Publications de l'université de Provence, 1998, p. 10.

- 13 - Nous n'évoquons pas ici toute l'activité annexe de la fédération, comme notamment l'organisation de trois congrès internationaux (1949 à Paris, 1950 à Bruxelles et 1951 à Amsterdam), elle sera évoquée plus loin dans la contribution de François Albera.

- 14 - Le Film sur l'art. Études critiques et catalogue international, Paris/Bruxelles, Unesco/Les Arts plastiques, 1949.

- 15 - Le Film sur l'art - Bilan 1950, études critiques, répertoire international, Paris / Bruxelles, Unesco/Les Arts plastiques, 1951. Cette publication connait une version en anglais sous le titre Films on art 1950 - a specialized study, an international catalogue (II), illustrée plus loin dans l'arcicle de Roxane Hamery.

- 16 - Bolen F. (dir.), Le Film sur l'art-Panorama 1953. Répertoire international illusiré, Paris/ Bruxelles, Unesco/Les Arts plastiques, 1953.

- 17 -- Ragghianti C. L. (dir.), Le Film sur l'art-Répertoire général international des films sur les arts, Rome, Edizioni dell'Ateneo, 1953. 
au même phénomène dans le champ anglophone et Outre-Atlantique, d'autant que la plupart des volumes édités par l'Unesco sur ce sujet sont publiés également en anglais. Ce mouvement se déploie donc avec des références comme William McKissack Chapman (dir.), Films on art 1952 (New York, American Federation of Arts, 1952) ou Theodore Robert Bowie, Films on art : a critical guide (Bloomington, Indiana, University Audio-Visual Center, 1956). Comme le révèlent leurs intitulés, ces catalogages ne se limitent pas à un simple recensement, mais s'habillent de bilans et d'études qui proposent un éclairage tout à la fois historique, critique et théorique sur ce "genre " qu'ils questionnent dans son nom (film sur l'art vs film d'art, en anglais art film vs films on art), son contenu, sa forme, ses fonctions et son évolution. Ces publications donnent ainsi au " genre " une réelle stature historiographique.

Cet essor bibliographique est en outre densifié par un autre type d'écrits qui fonctionnent un peu comme des " compléments " aux films. En effet, la plupart des films sur l'art qui émergent alors sont d'un tout nouveau genre - en phase avec le renouveau "formaliste " de l'histoire de l'art de l'époque -, usant du cinéma à des fins analytiques, comme un langage visuel nouveau qui s'adresse à l'œil du spectateur. La production conjointe de textes est ainsi souvent envisagée comme un moyen d'expliquer, promouvoir, justifier, ou « reformuler » verbalement le discours mis en place et expérimenté « cinématographiquement ${ }^{18}$ ». Ces films analytiques convoquent le plus souvent des historiens de l'art et ceux-ci, habitués à prendre la plume, n'hésitent pas à accompagner leur travail filmique de publications écrites qui développent ou expliquent ce nouvel usage "scientifique " de la caméra dans l'analyse des œuvres. Le plus célèbre est sans aucun doute Ragghianti, qui, entre 1948 et 1964, concrétise par la réalisation de vingt-et-un « critofilms » sa théorie du cinéma comme moyen privilégié de décrypter les ouvres et de faire de l'histoire de l'art non par les mots, mais par l'image - théorie qu'il synthétise également par écrit dans Cinema arte figurativa (Turin, Einaudi, 1952) puis, plus tard, dans le premier volume de son recueil Arti della Visione (Turin, Einaudi, 1975) ${ }^{19}$. Paul Heilbronner, écrivain allemand élève de Wölfflin, fera l'inverse, en " précurseur ", puisqu'avant de réaliser des films sur l'art aux États-Unis sous le nom de Laporte, il émigre d'abord en Italie et y écrit plusieurs textes préconisant de filmer les œuvres d'art - peut-être en s'inspirant de Ragghianti, une polémique existe à ce sujet tels que "Cinema documentario : il cinema e le belle arti » dans Cine-convegno

- 18 - L'un des meilleurs exemples, dans le champ français, est probablement le n 37-38-39 (1949) de L'Amour de l'art (revue d'art dirigée par Germain Bazin, alors conservateur au musée du Louvre), entièrement consacré à la question des films sur l'art (il se conclut d'ailleurs sur un " répertoire international » de ces films), avec des textes de Jean Cocteau, Paul Haesaerts, Gaston Diehl, etc. - 19 - Voir à ce sujet Raynaud P., "Voir et savoir. Les critofilms de Carlo L. Ragghianti ", in Le Forestier L. et Mouëluic G. (dir.), Filmer l'artiste au travail, op. cit., p. 289-308. 
- 18 - F. Albera, L. Le Forestier, V. Roberi, K. Imesch ef M. Lüscher

$\mathrm{n}^{\circ} 3-4-5$ (1934), ou «Il cinema come arte figurativa » dans Intercine, $\mathrm{n}^{\circ} 7$ (1935) ou encore "Cinematografando le opere d'arte », Cinema, n 4 (1936).

Mais la discussion va surtout éclore " en aval ", dans le champ de la réception critique. Aussi bien dans La Revue du cinéma que dans L'Écran français, ces films d'un nouveau genre connaîtront en effet un retentissement immédiat, avec des articles tels que "Les Origines de la mise en scène " de Jean George Auriol (La Revue du cinéma, $\mathrm{n}^{\circ} 1,1946$ ), paru en même temps que "Paroles peintes" de Piero Bargellini (ibid.) ou "Quand la caméra "explore” les chefs-d'œuvre de la peinture "signé par Pierre Kast ${ }^{20}$ (L'Écran français $\mathbf{n}^{\circ} 55$, 1946). Ces textes voient dans « l'assimilation » qu'opère le film sur l'art la « preuve » d'une communauté de langage entre le cinéma et les arts plastiques, confirmant que le cinéma est un «art

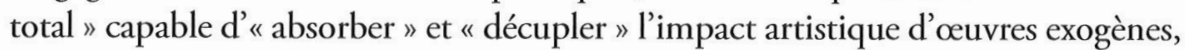
et incitant à considérer certaines peintures comme "pré-cinématographiques" - adhérant donc complètement au discours filmique qui, tel Racconto da un'affresco (Luciano Emmer et Enrico Gras, 1940), ne nous dit rien d'autre que... " Giotto faisait du cinéma "! Si certains textes accuseront ces films de "profanation artistique » capable de dégrader la "poésie dense et solennelle» des fresques de Giotto en "petit roman de gare pour écolières " ${ }^{21}$, ce ne sera pas au détriment du genre ni de sa fortune critique, puisqu'ils loueront en revanche des films sur l'art d'un autre type, et ouvriront la voie aux développements plus didactiques de cette production - ainsi Umberto Barbaro réalisera avec Roberto Longhi le film Carpaccio, suivant une méthode analytique mise au service, non pas du drame, mais de l'histoire de l'art, de l'étude scientifique de l'œuvre. Ces élans critiques accompagnent et relancent donc l'expansion filmographique, et se cristallisent aussi en ouvrages plus autonomes, comme la brochure Le belle arti e il film éditée à l'occasion de la onzième Mostra internazionale d'arte cinematografica di Venezia (Rome, Bianco e nero, 1950), comme la publication, dans la revue Film, des actes du colloque qui, à Florence en 1955, a fait le point sur « le cinéma et les arts figuratifs ${ }^{22}$ », enfin comme l'ouvrage d'Henri Lemaître Beaux-Arts et cinéma (Paris, Les Éditions du Cerf, 1956). Ces ouvrages tissent des bilans historiques et critiques sur le film sur l'art, qu'ils considèrent comme la "preuve " des " affinités ", " continuités ", " échanges " et "synthèses " qui lient cinéma et arts plastiques. Pour Henri Lemaître, le film sur l'art, où " l'œuvre cinématographique v[ient] en quelque

- 20 - Pierre Kast a lui-même co-réalisé (avec Jean Grémillon) des documentaires sur l'art comme Les Charmes de l'existence (1950) et Les Désastres de la guerre (1951) et, seul, L'Architecte maudit: Claude-Nicolas Ledoux (1954).

- 21 - Barbaro U., "Critica e arbitrio nel documentario sulle arti figurative ", Bianco e Nero, no 8-9, 1950, p. 47-52.

- 22 - « II convegno sul cinema e le arti figurative " [1956], Film (Florence), n 5-6. 
sorte se superposer organiquement aux œuvres qui lui servent de sujet ${ }^{23}$ ", révèle non seulement combien - depuis ses "premières origines ${ }^{24}$ " - «l'art des images gravées, dessinées ou peintes » est l'expression d'un « langage déjà virtuellement cinématographique ${ }^{25}$ ", mais ce nouveau genre marque surtout la "naissance d'une cinécritique " ou d'une " ciné-histoire ${ }^{26} "$ qui actualise des affinités entre le cinéma, "art de l'ubiquité " et de la "confrontation ", et l'histoire de l'art. Au point que selon Lemaître, «le film sur l'art accomplit et achève le vœu du "Musée imaginaire” » de Malraux :

"Le Musée imaginaire [...] fait appel à une technique de la photographie où déjà apparaissent des méthodes cinématographiques, et les meilleurs "livres d'art" sont souvent ceux où le choix des illustrations, leur cadrage, leur éclairage, leur agencement, sont déjà comme l'amorce du découpage et du montage d'un éventuel film sur l'art. [...] Entre le cinéma et le Musée imaginaire, il y a plus qu'une analogie formelle : [ils] modifie[nt] radicalement à la fois l'image elle-même et les structures de notre vision ${ }^{27}$. "

\section{«Spécificité » bazinienne ef kracauérienne}

De part et d'autre de l'Atlantique, deux critiques et théoriciens influents contribuèrent à la réflexion sur le film sur l'art à partir des spécificités du médium filmique : André Bazin et Siegfried Kracauer. Le paradoxe veut que l'un et l'autre aient été les partisans d'une définition du cinéma comme "ontologiquement " réaliste ou, pour reprendre les termes mêmes de Kracauer, attachée au cinéma «se conformant à l'affinité du médium pour le matériau naturel ${ }^{28}$ ". Or c'est précisément cette extériorité du monde de l'art - tableau, dessin sculpture - au «matériau naturel» qui les conduit, par des voies différentes, à considérer qu’une fois filmée l'œuvre d'art en vient à « apparaître comme un élément de la réalité2 ${ }^{29}$ ». Les décalages entre les époques où ils découvrent ces films, la disponibilité de ceux-ci et les interlocuteurs différents qui sont les leurs, font que leurs réflexions

- 23 - Lemaître H., Beaux-arts et cinéma, Paris, Les Éditions du Cerf, 1951, p. 78.

- 24 - Lemaître fait ici référence aux " dessins gravés sur la pierre de Lascaux ou d'Altamira " que filme William Chapman dans Lascaux, Cradle of Man's Art (1950). Ce topos est régulièrement relancé, ainsi très récemment par le préhistorien Marc Azéma (La Préhistoire du cinéma. Origines paléolithiques de la narration graphique et du cinématographe... Préfaces de Jean Clottes et Bertrand Tavernier Paris, Éditions Errance, 2011).

- 25 - Lemaître H., op. cit., p. 12.

- 26 - Ibid., p. 137. À cet égard Ragghianti accusa Lemaître d'avoir plagié ses théories.

- 27 - Ibid., p. 128-129.

- 28 - Kracauer S., Théorie du film. La rédemption de la réalité matérielle, Paris, Flammarion, 2010 [1960], p. 289.

- 29 - Ibid. 
F. Albera, L. Le Forestier, V. Robert, K. Imesch er M. Lüscher

ne s'appliquent pas aux mêmes objets ou ne mettent pas l'accent sur le même point quand ils coïncident : tous deux voient et commentent Le Mystère Picasso de Clouzot, mais le Français Bazin s'intéresse avant tout aux films de Resnais - les autres films servant de repoussoirs ou d'éléments de comparaison -, tandis que l'émigré allemand aux États-Unis Kracauer tente d'embrasser l'ensemble du phénomène, partant du Michel Ange d'Ertel, du Goya d'Emmer, du Monde de Paul Delvaux de Storck, jusqu'au Henry Moore de John Read, au Works of Calder de Herbert Matter ou aux films de Hans Richter. Pour Kracauer dès lors qu'il est filmé - c'est-à-dire fragmenté, mis en mouvement - le tableau se trouve délié de la " composition artistique " à laquelle il appartenait et revêt "l'apparence de la vie telle qu'elle se déploie hors de la dimension esthétique ${ }^{30}$ ». Mais pour lui ce processus tient avant tout aux rapports de l'écran et du spectateur : ce sont les " attentes » de celui-ci qui dote de "l'indétermination de la vie réelle " les " entités détachées » (gros plans, détails) que le cadrage prélève sur l'œuvre artistique, son imagination complétant cette « existence fragmentaire en l'insérant dans des contextes de [son] choix ». En outre le mouvement de la caméra suscite des "réactions cinesthésiques -- "effet de résonance" qui conduit le spectateur à projeter les sensations spatiales qu'il ressent de ce fait dans ses perceptions simultanées ${ }^{31}$ " faisant « gagner [l'image] en profondeur spatiale » et permettant une participation au spectacle proposé ${ }^{32}$. Kracauer par ailleurs distingue le « film expérimental » du " courant documentaire ». Le premier part du tableau ou de la sculpture comme d'un «matériau brut qu'il façonne selon ses propres visions " ne cherchant ni à le restituer, ni à mettre en lumière ses qualités intrinsèques. Il agit selon la procédure, courante en art, du «transfert ». Pour lui : «Le film expérimental sur l'art se réalise pleinement lorsque son auteur, indifférent à toute visée étrangère, s'efforce de construire, à partir des éléments que comportent les tableaux utilisés, une totalité qui se suffit à elle-même et qui a autant de valeur artistique que l'original. » Cet " objet filmique autonome qui prétend à son tour être de l'Art » appartient pour lui plutôt à l'art contemporain qu'au cinéma : "c'est ailleurs que se situe le centre de gravité du médium ${ }^{33}$. "Cet ailleurs c'est le " courant documentaire » qui s'en approche en s'intéressant à la genèse de l'œuvre d'art plutôt qu'à l'œuvre elle-même et en particulier il permet au spectateur d'être « témoins du processus créatif qui

- 30 - Ibid., p. 285.

- 31 - Ibid., p. 284-285. Cette attention au spectateur qui « complète » l'image, réagit aux procédés mis en ouvre témoigne de l'intérêt que porte Kracauer aux recherches et aux expérimentations menées dans le cadre de I'Institut de filmologie à Paris.

- 32 - « [II] se verra lui-même fânant au creux de la vallée que montre la peinture » (ibid., p. 285).

- 33 - Ibid., p. 288-289. Kracauer qui vit à New York est en contact avec les groupes de cinéastes et de critiques expérimentaux qui gravitent autour de la revue Film Culture créée par Jonas Mekas - à laquelle il a collaboré. 
l'engendre » : soit, comme dans Le Mystère Picasso, en nous permettant « d'assister à l'acte créateur ", soit par le montage par surimpression d'esquisses produisant "l'illusion d'une évolution organique ${ }^{34}$ ".

André Bazin de son côté participe à la fortune critique du film sur l'art très tôt en commentant Rubens (Haesaerts et Storck, 1948) ou Van Gogh (Resnais, 1948) ${ }^{35}$, et lui réservera un traitement particulier dans son recueil Qu'est-ce que le cinéma (Paris, Les Éditions du Cerf, 1958). Là où d'autres voient dans le film sur l'art la preuve d'une identité expressive entre cinéma et arts plastiques, Bazin s'attache moins aux ressemblances qu'aux décalages apportés par cette « cinématisation » de la peinture. Le film sur l'art lui permet de développer une théorie du cadrage basée sur l'opposition entre le cadre du tableau et le cadre - ou plutôt le " cache »-de l'écran : le premier, «centripète », conditionnerait une organisation de la représentation « en dedans », circonscrivant l'espace au tableau, tandis que le deuxième, "centrifuge ", impliquerait toujours un hors-champ, organisant sa représentation de manière " ouverte ", comme une simple "partie " d'une réalité spatiale dépassant l'écran. Mais Bazin ne limite pas à cette définition différenciée du cadre, son chapitre "peinture et cinéma » contient le texte intitulé « Un film bergsonien : Le Mystère Picasso », qui propose l'une des premières réflexions théoriques sur les enjeux spécifiques de la représentation de l'acte pictural ${ }^{36}$. Le Mystère Picasso de Clouzot (1956) a, en effet, cette « audace extraordinaire » d'être entièrement construit par le filmage, non de peintures achevées, mais de peintures en création; Bazin y voit un bouleversement artistique et théorique qui fait appel à la « spécificité » du cinéma, assimilant la " contemplation de l'ouvre en création, du work in progress " comme « l'élément spectaculaire authentique, c'est-à-dire cinématographique, parce qu'essentiellement temporel $^{37}$ ", pour dévoiler une "spécificité » picturale :

"Ce que révèle Le Mystère Picasso, ce n'est pas ce que l'on savait déjà, la durée de création, mais que cette durée peut être partie intégrante de l'œuvre même, une dimension supplémentaire, bêtement ignorée au stade de finition. Plus exactement, nous ne connaissions jusqu'ici que "des tableaux", sections verticales d'une coulée créatrice plus ou moins arbitrairement tranchée par l'auteur lui-même, par le hasard, par la maladie

- 34 - Tyler P., "The Film Sense and the Painting Sense », Art Digest, 15 février 1954 (cité par Kracauer, op. cit., p. 292).

- 35 - Bazin A., "Le cinéma et la peinture : À propos de Van Gogh et Rubens ", La Revue du cinéma, $2^{\mathrm{e}}$ série, $\mathrm{n}^{\circ} 19-20,1949$, p. 114-119.

- 36 - Pour une étude des formes et enjeux de la représentation cinématographique de l'acte de peindre, voir Roвert V., "Filmer peindre. La représentation filmique de l'acte pictural, miroir / faire-valoir de l'acte cinématographique " (université de Lausanne, 2006).

- 37 - Bazin A., "Un film bergsonien : Le Mystère Picasso (1956) ", Qu'est-ce que le cinéma, Paris, Les Éditions du Cerf, 1959, vol. 2, p. 137. 
ou la mort. Ce que Clouzot, lui, nous révèle enfin, c'est "la peinture", c'est-à-dire un tableau qui existe dans le temps, qui a sa durée, sa vie, et quelquefois - comme à la fin du film - sa mort ${ }^{38}$."

L'historiographie de l'art a, en un certain sens, emboîté le pas à Bazin, en se renouvelant grâce à un film sur l'art donnant à voir dans le temps le geste d'un artiste. Mais ce ne fut ni par ce film ni par cet artiste. En effet, malgré sa très bonne réception dans le champ cinématographique (dont témoigne le prix du jury de Cannes), et la manière dont, "plus qu'aucun autre facteur ${ }^{39}$ ", il rendit Picasso accessible au grand public, le film de Clouzot connut un accueil réservé de la part des critiques d'art à sa sortie : John Berger notamment condamna la mise en scène du réalisateur pour dénaturation de l'œuvre picassienne ${ }^{40}$, d'autres, comme Hélène Parmelin, parlant d'infidélité ${ }^{41}$. De nos jours cependant l'image de Picasso devant sa toile filmé par Clouzot en est venue fréquemment à incarner " par excellence » la figure de l'artiste-créateur. La couverture du numéro de la revue Communications consacrée en 1997 à "La création " (sous la direction de l'anthropologue François Flahaut et de l'esthéticien Jean-Marie Schaeffer) en donne un photogramme où Picasso de dos, jambe fléchie, dans un éclairage "expressionniste ", semble sur le point de bondir sur la toile qui lui fait face. La "promotion de la figure du créateur est allée de pair avec une dévaluation de l'œuvre et plus généralement de l'art comme activité productrice d'objets ${ }^{42}$ " écrivent les directeurs et l'un reprend plus loin l'exemple du film de Clouzot où « l'accent est mis sur l'acte de création [...] plutôt que sur le résultat ".

\section{L'inspiration de l'action}

Un peu auparavant paraissait sur le continent américain les films et les séries photographiques de Hans Namuth sur Jackson Pollock, qui allaient avoir un impact profond dans l'historiographie, non du cinéma mais de l'art. Les captations de Namuth sont en effet à l'origine de la définition et de la reconnaissance de

- 38 - Ibid., p.135-136.

- 39 - Hollowny M. J., Making Time: Picassós Suite 347, New York, Peter Lang, 2006, p. 16.

- 40 - Berger J., "Clouzot as Delilah ", Sight and Sound, vol. 27, n 4, 1958; PArmelin Hélène,

"Le peintre et la caméra ", Notre-Dame de Vie, Paris, Cercle d'Art, 1966.

- 41 - Parmei.jn H., "Le peintre et la caméra ", Notre-Dame de Vie, op. cit., p. 18-19.

- 42 - Flahnut F. et Schaeffer J.-M., "Présentation ", Communications, no 64, 1997, p. 5. Les auteurs rattachent le postulat, " tacitement admis dans les secteurs les plus radicaux de l'art contemporain ", selon lequel "l'identité de l'art réside dans son concept et plus généralement dans sa signification en sorte que l'objet sensible importe uniquement comme support de cette idée et non comme source de délectation esthétique propre" au romantisme philosophique qui fait de l'artiste * l'incarnation modèle du sujet humain autofondé et fondateur du monde " (p. 10-11). 
l'action painting. Les deux films, l'un en noir-blanc et l'autre en couleur connu sous le titre Jackson Pollock 51 (Hans Namuth et Paul Falkenberg, 1951), ainsi que les séries photographiques qui montrent le peintre dans son atelier ont révélé cette dimension créatrice, temporelle et vivante de l'art qui émerveillera Bazin et qu'on pourrait appeler la "peinture en action». Ces images documentaires auront un tel impact dans l'historiographie de l'art que - dès notamment les fameux essais (assimilés même à des manifestes) de Robert Goodnough, "Pollock Paints a Picture ", paru en mai 1951 dans Art News (la revue décidant précisément d'illustrer l'article avec des photos de Namuth) et " The American Action Painters " de Harold Rosenberg qui paraît dans la même revue l'année suivante (décembre 1952) - l'ensemble de la littérature sur Pollock semble moins prendre pour sujet sa peinture que les captations de son geste par Namuth ${ }^{43}$.

L'historienne de l'art Barbara Rose a montré combien, en révélant « l'acte - le processus de création artistique - au lieu de l'objet statique », les images de Namuth, " avec une ampleur qu'il ne pouvait lui-même ni prévoir ni imaginer ", ont " changé le cours de la critique d'art, et même de l'histoire de l'art ${ }^{44}$ ". Si ce constat est évidemment rétrospectif, dès 1958 des contributions telle celle de l'artiste Allan Kaprow dans Art News ("The Legacy of Jackson Pollock ») pressentent les relais et développements théoriques qu'auront ces photographies et ces films sur Pollock, qui vont inspirer toute une génération d'artistes et une nouvelle conception de l'art, fondée sur la performance ${ }^{45}$. Le statut du film sur l'art va se voir transformé par cette acception "performative " de l'œuvre, à laquelle il pourra désormais participer - la captation devenant le moyen, sinon d'expression, du moins de survivance des performances. Corrélativement, le statut du réalisateur de film sur l'art prendra une nouvelle dimension, en concurrence directe avec l'artiste filmé - en témoigne par exemple l'exposition du travail photographique de Namuth à la célèbre Stable Gallery de New York, dans un accrochage (Photographs of Seventeen American Painters, 1958) qui a même représenté les États-Unis à l'Exposition universelle de Bruxelles. Ce renouveau théorique va ainsi susciter, par jeu de réciprocité, ce que Paola Scremin a identifié comme une recrudescence du « film sur l'art processuel » dans les années $1960^{46}$, tandis qu'en

- 43 - Rose B., « Hans Namuth's Photographs and the Jackson Pollock Myth. Part I: Media Impact and the Failure of Criticism ", Arts Magazine, vol. 53, n 7, mars 1979, p. 112-119.

- 44 - Rose B., Pollock Painting. Photographs by Hans Namuth, New York, Agrinde Publications, 1980, p. 1941.

- 45 - Voir le bilan de cette influence que dresse la rétrospective « Pollock " qui s'est tenue au MoMA en 1998 et tous les textes qui sont parus a cette occasion, notamment celui de Boxer S., "The photos that changed Pollock's Life ", The New York Times, 15 décembre 1998.

- 46 - Scremin P., «Film sull'arte ", Enciclopedia del Cinema, Treccani.it, 2003, URL : http:// www.treccani.it/enciclopedia/film-sull-arte_\%28Enciclopedia-del-Cinema\%29/. L'expression est 
- 24. - F. Albera, L. Le Forestier, V. Robert, K. Imesch el M. Lüscher

anglais ce type de documentaires sur le work in progress a plus volontiers été appelé "visite d'atelier ${ }^{47}$ " ou « artiste au travail ${ }^{48}$ ». Elle mentionne par ailleurs d'autres facteurs de développement d'un tel « genre » cinématographique, ressortissant au champ de l'histoire du cinéma (et non uniquement de celle de l'art), tel que la politique du " cinéma-vérité " ou l'établissement de la télévision ${ }^{49}$.

\section{Dernières pages?}

Le soutien bibliographique et institutionnel du film sur l'art est encore actif dans les années 1960. L'entreprise de catalogage générique se poursuit avec des publications dans le cadre de la FIFA, telles que Dix ans de films sur l'art (19521962): Catalogue (volume 1 : «Peinture et sculpture », Paris, Unesco, 1966, avec une contribution introductive d'Henri Lemaître) ou Le Film sur l'art. Répertoire général international du film sur l'art 1953-1960 dirigé par Pasquale Rocchetti et Cesare Molinari (Vicence, Neri Pozza, 1963). Une structure comme le " Centre canadien du film sur l'art " voit même le jour à Ottawa - suite au Festival et Colloque de films sur l'art organisé à la Galerie nationale par Dorothy MacPherson en 1963 - et garantit la survivance de publications et de répertoires. Le centre maintiendra le catalogage universel en éditant (Ottawa, Canadian Centre for Films on Art) en 1968 le Catalogue des films sur l'art, en 1973 Films on art 10 : catalogue et en 1977 Films on art: a source book. Mais ce catalogue de 1977, qui d'ailleurs est financé grâce à l'American Federation of Arts et co-édité à New York chez Watson-Guptill, sera le dernier : le centre s'éteint ensuite, après une période de

de Philippe-Alain Michaud pour qui il s'agit là de films « accompagn[ant] visuellement le processus créateur en utilisant les ressources de la caméra " (Мichaud P.-A., "Le film sur l'art a-t-il une existence?" "in Chevrefils Desbiolles Y. [dir.], Le Film sur l'art et ses frontières, op. cit., p. 15-16). - 47 - Cover't N. et Montgomery H., "Art on Screen : film and television on art - an overview ", in Covert N. et Montgomery H. (dir.), Art on Screen: A Directory of Films and Videos About the Visual Arts, New York, The Metropolitan Museum of Art, 1991, p. 3.

- 48 - Wechsler J., "Art History and Films on Art ", ibid., p. 9.

- 49 - Scremin P., op. cit. Notons que René Rozon a vu dans ces mêmes facteurs les origines d'un développement du film sur l'art allant du côté du reportage bien plus que du film processuel ; « Les moyens de communication, en particulier la télévision, ont envahi nos vies. [...] Lartiste et son travail ne suffisent plus à satisfaire le spectateur. Il faut l'attirer par d'autres moyens. Et la meilleure façon est de dévoiler sa vie personnelle. On s'attarde à sa vie sentimentale, à ses difficultés financières, à ses contacts professionnels notamment. L'artiste ne se cache plus derrière son œuvre, il est mis à nu. Le cinéma direct a bien servi. Rappelons A Bigger Splash de Jack Hazan, portrait de David Hockney. Est venue s'ajouter, par la suite, une nouvelle dimension : le contexte sociopolitique. En tentant de replacer l'artiste dans son milieu, de rappeler les circonstances qui l'ont marqué, avec incursion psychanalytique parfois " (Rozon René, "Flash-back ", $25^{e}$ Festival international du film sur l'art: Répertoire, Montréal, FIFA, 2007, p. 3). 
précarité qui aura duré en tout cas tout au long des années $1970^{50}$ - la dissolution de la FIFA remontant à 1970 même.

On doit en effet constater une chute de l'intérêt historiographique pour ces films dans les années 1970 : le film sur l'art ne suscite plus de commentaire, ni même d'attention, au point qu'on puisse déplorer qu'il « [soit] certainement, de tous les genres cinématographiques, le plus méprisé et le plus méconnu, car il est le plus souvent assimilé au documentaire didactique ennuyeux et poussiéreux ${ }^{51}$ ".

\section{Festivall d'écrits}

Dans les années 1980 une réactualisation historiographique du sujet s'opère mais par un autre versant, celui de la relation entre cinéma et peinture ainsi qu'avec l'avènement de festivals y consacrés.

Cet intérêt voire cette fascination de théoriciens, esthéticiens et philosophes pour le lien entre beaux-arts et cinéma - dont il serait trop long d'évoquer ici les multiples publications, colloques et numéros spéciaux de revues ${ }^{52}$ - ramènera les films sur l'art dans l'actualité, d'autant que des festivals vont permettre à cette production de retrouver un ancrage critique. Parler de " renaissance » du champ d'études n'est sans doute pas exagéré puisque succédant à la FIFA (fondée en 1949 à Paris) qui disparaît en 1970, le FIFA (Festival international du film sur l'art) naît en 1981 à Montréal, et connaît un grand succès jusqu'à nos jours ${ }^{53}$, tandis que d'autres festivals de films sur l'art se sont multipliés en tous pays jusque très récemment, et surtout en Italie. Citons ainsi le Festival Internazionale del Film sull'Arte e di Biografie d'Artista lancé à Trévise en 1973, qui disparaît puis renaît en 2001, réorganisé sur des bases institutionnelles et renommé AsoloArtFilmFestival, le Festival internazionale del Cinema d'Arte créé à Bergame en 2002, déplacé au Palais Royal de Milan dès 2013, sans compter les quatre éditions du Festival d'Arte

- 50 - Rozon R., « Dorothy Macpherson ou la démocratisation de l'art par le film ", Vie des Arts, vol. 23, no 91,1978 , p. 63-93.

- 51 - Bassan R., "Art et cinéma ", Écran, n 53, 1976, p. 6. Soulignons que, si l'historiographie n’en a plus parlé, la production ne s'est jamais " tarie ", mais seulement " déplacée ". Car, avec la diffusion et les perfectionnements colorisés de la télévision dans les années 1960, les films sur l’art, demandés et subventionnés par les chaînes qui promeuvent les émissions culturelles, se sont alors spécialisés dans le format télévisuel, le plus souvent didactique et traditionnel.

- 52- Pour un bilan historiographique détaillé, qui culmine avec des monographies spécialisées telles celles de Jacques Aumont, Antonio Costa, Brigitte Peucker, Angela dalle Vacche ou John A. Walker, voir Robert V., "Introduction. Fictions de création, la peinture en abyme et le cinéma en question ", in Le Forestier L. et Mouëllic G. (dir.), Filmer l'artiste au travail, op. cit., p. 19-24.

- 53 - www.artfifa.com. Notons qu'il y eut, avant sa naissance "officielle ", des éditions un peu informelles de ce festival, la première datant de 1976, à bord du "Mermoz ", dont Bassan fait précisément état dans son article d'Écran, art. cit. 
- 26 - F. Albera, L. Le Forestier, V. Robert, K. Imescher M. Lüscher

di Palazzo Venezia, qui eut lieu à Rome de 2004 à 2007, en tant que festival international des films et des documentaires sur l'art.

Le festival est un agent d'élaboration critique privilégié : faisant acte de regroupement, recensement, présentation et débat (tant par écrit que par oral), il inscrit par principe sa sélection de films sur l'art dans une historiographie, en même temps qu'il lui offre l'occasion de publications critiques dans la presse généraliste et spécialisée ${ }^{54}$. Les catalogues de ces manifestations, souvent enrichis de commentaires critiques ou historiques généraux, sont des contributions importantes pour l'histoire du genre - qu’ils construisent. Ainsi du répertoire quinquennal édité par René Rozon à l'occasion du $25^{\circ}$ Festival international du film sur l'art de Montréal en $2007^{55}$, et des catalogues qui ont accompagné trois des six éditions de la Biennale internationale du film sur l'art dirigée par Gisèle Breteau-Skira au Centre Georges Pompidou de 1987 à 1998, avec cette particularité d'adopter des axes thématiques: Picasso à l'écran (1992); $5^{e}$ Biennale du film sur l'art (autour du thème de la nuit) (1996) et Ge Biennale du film sur l'art (autour du thème du désert) (1998). Des rétrospectives ont d'ailleurs eu lieu ponctuellement, avec des publications tout aussi pertinentes, voire davantage, comme en témoigne la publication éditée par Anne Coutinot en accompagnement de la rétrospective de films sur l'art organisée à Paris par le ministère des Affaires étrangères en mars 1992, qui se présente comme une anthologie saisissante de textes sur le film sur l'art, faits à partir d'extraits théoriques et d'entretiens avec des artistes filmés et des cinéastes filmeurs ${ }^{56}$.

Bien d'autres événements ou publications témoignent de cette résurgence d'intérêt depuis les années 1980, des deux côtés de l'Atlantique. Citons notamment la fondation en 1980 en Belgique du Centre du film sur l'art (CFA) qui constitue aujourd'hui une véritable cinémathèque spécialisée, répertoriant, documentant et mettant à disposition près de deux cents films sur l'art; ou encore la fondation, en 1984, du Program for Art on Film, au Metropolitan Museum of Art de New York, qui, en plus de la publication papier dirigée par Nadine Covert de Art on screen: a directory of films and videos about the visual arts, (New York, Program for art on film, 1991), met en ligne une base de donnée listant à ce jour plus de 25000 films sur l'art (www.artfilm.org). En France, on n'a pas repéré de base de données

- 54 - Alain Fleischer a par ailleurs soulevé la différence d'attention que suscite la diffusion festivalière des films sur l'art, ainsi " extraits » du petit écran de télévision : "la projection des films sur grand écran leur redonne une dimension perdue, et la salle de cinéma rétablit l'ambiance collective et festive du spectacle, imposant la règle de sa cérémonie " tout en permettant de "voir des versions hors-format, inédites, plus longues et plus libres que celles imposées par les industries télévisuelles ". Fleischer A., "Au FIFA, le film sur l'art en grandeur réelle ", $25^{e}$ Festival international du film sur l'art: Répertoire, op. cit., p. 4.

- 55 - Rozon R., «Flash-back ", ibid., p. 3.

- 56 - Coutinot A. (dir.), Peinture et cinéma, Paris, ministère des Affaires étrangères, 1992. 
comparable, mais on peut signaler l'édition, en 1985, d'un inventaire véritablement encyclopédique des films sur l'art dirigé par Gisèle Breteau-Skira, l'Abécédaire des films sur l'art moderne et contemporain : 1905-1984 dont Dominique Bozo a poétiquement dit qu'il était «la première cinémathèque imaginaire, l'architecture sans fin des désirs, des regards, des curiosités et des raisons, de tous les témoins de l'art du xx ${ }^{\mathrm{e}}$ siècle, ceux nommément indexés ici ${ }^{57}$ ". D'autres entreprises d'envergure sont à citer, comme l'inventaire de la Roland Collection of Films on Art publié en $1996^{58}$ et devenu, grâce à Internet, une base de données mettant à disposition sous forme d'extraits plus de 400 films sur l'art (www.rolandcollection.com), et surtout la dernière publication du Program for Art on Film du MoMA, qui consiste en un répertoire historiographique du film sur l'art, intitulé Films on art : bibliography (New York, Program for Art on Film, 1994).

\section{Une double place (théorique) à prendre}

Mais les publications théoriques récentes sur le film sur l'art restent extrêmement rares. On ne peut guère citer que deux ouvrages spécialisés, celui de Fanny Étienne, Films d'art/Films sur l'art (Paris, L'Harmattan, 2002) et surtout l'essai de Gilles Marsolais, Le Film sur l'art et le cinéma : fragments, passages (Montréal, Tryptique, 2005) qui éclairent les différentes formes et sous-genres de cette production par des analyses qui en révèlent -- sans les dénouer - les complexités et multiplient les fils conducteurs. Quant aux entreprises collectives, il y eut Zeuxis, revue critique spécialisée sur le film sur l'art (envisagé dans une acception large et grand public) lancée par la même Gisèle Breteau-Skira en 2000 - trente-trois numéros couronnés par l'édition, en 2010, d'une anthologie d'entretiens que la revue avait réalisés avec des réalisateurs de fictions sur l'art. Les colloques qui se concentrèrent réellement sur la question du film sur l'art sont rares. Hormis "Filmer l'acte de création » qui, sous la direction de Pierre-Henri Frangne, Gilles Mouëllic et Christophe Viart à Rennes en avril 2007 (publié en 2009), leva le voile sur la richesse de cette problématique générale qu’est « filmer l'art " et fut comme un point de départ aux développements plus spécifiques qu’on a évoqués ${ }^{59}$, on

- 57 - Bozo D., "Abécédaire : pari plausible ", dans Gisèle Breteau, Abécédaire des films sur l'art moderne et contemporain : 1905-1984, Paris, Centre Georges Pompidou/Centre national des arts plastiques, 1985 , p. 6.

- 58 - ROLAND A., Videos on art: a resource guide to films and videos available worldwide from the Roland Collection, Harriman (N.Y.), Roland Collection of Film on Art, 1996.

- 59 - Le projet de recherche «Filmer la création " (FILCREA) a reçu le soutien de l'Agence nationale de la recherche, ce qui a permis au laboratoire de cinéma de l'université Rennes 2 d'organiser de nombreuses journées d'études et plusieurs colloques sur le sujet. À ceux déjà mentionnés, on peut ajouter le colloque "L’œuvre de l'art. La pensée esthétique de Gérard Genette " (sous la direction 
ne peut guère citer qu'un seul autre colloque, qui tenta une même approche théorique multiple du genre, et qui eut lieu il y a quinze ans, en mars 1997, à Aix-en-Provence, sous la direction d'Yves Chevrefils Desbiolles, intitulé Le Film sur l'art et ses frontières (actes publiés par l'université de Provence un an après). Le questionnement demeurait donc ouvert - et nécessaire, ainsi qu'en témoigna Thierry Dufrêne quelques mois avant l'ouverture notre colloque, déplorant la rareté des "recherches menées en réseau sur les rapports de l'histoire de l'art et du film ${ }^{60}$ ». Il intimait aux chercheurs de mener une réflexion sur "l'histoire de l'art à l'âge du cinéma ${ }^{61}$ ", impliquant tout à la fois de " mesurer l'impact de l'audiovisuel (englobant le cinéma) sur les contextes où l'histoire de l'art s'exerce : la recherche, le musée, l'enseignement ${ }^{62}$ ", d'analyser « la manière dont le cinéma s'est emparé, au cours de son histoire, des œuvres d'art ${ }^{63}$ " et de " comprendre [au cour de la discipline de l'histoire de l'art] comment notre vision du temps historique a été affectée par le cinéma ${ }^{64}$ ». Les contributions de ce volume rencontrent très étroitement ces enjeux, en se concentrant sur l'objet d'étude qui, par excellence, les cristallisent : les « films sur l'art », ces « documentaires de création " que Dufrêne rebaptise " films d'histoire de l'art ${ }^{65}$ ".

\section{Et l'histoire de l'art?}

En dépit de ces développements du genre, de la régularité de sa production et des présentations qu'on en fait, force est cependant de reconnaître que ni le cinématographe ni ses avatars n'ont transformé le rapport des historiens de l'art à leurs objets et, par là, à leur discipline, à l'égal de ce qu'avait opéré la photographie. Celle-ci documentait plus finement que ne le pouvait le regard (détails agrandis, éclairage de zone d'ombre, accès à des parties inaccessibles de l'architecture, possibilité de confronter les images, de les superposer, de les classer) et, bien qu'elle transformât profondément la nature de l'objet étudié - irréalisé en quelque sorte quant à sa nature matérielle au profit d'une saisie en terme d' ' image " que l'ère numérique accomplit définitivement (avec l'assentiment de certains à ce

de Pierre-Henry Frangne, Gilles Mouëllic et Joseph Delaplace, les 25, 26 et 27 novembre 2010). Par ailleurs, ce vaste projet de recherche a débouché sur plusieurs publications, parmi lesquelles on peut citer : Thivat P.-L. (dir.), Biographies de peintres à l'écran, Rennes, Presses universitaires de Rennes, 2011, et Le Forestier L. et MovëLlic G. (dir.), Filmer l'artiste au travail, op. cit.

- 60 - Dufrêne T., « L'histoire de l'art à l'âge du cinéma », Diogène, $\mathbf{n}^{0}$ 231, 2010/3, p. 147.

- 61 - Ibid., p. 137.

- 62 - Ibid., p. 143.

- 63 - Ibid., p. 145.

- 64 - Ibid., p. 146.

- 65 - Ibid., p. 137. 
processus) -, elle paraissait maximiser la vue du spectateur ordinaire comme le fait une loupe ${ }^{66}$, une lunette ou un microscope et non proposer une procédure nouvelle ${ }^{67}$. Le film sur l'art, dans son ambition la plus grande, aurait pu permettre autre chose : la vision en mouvement, la redistribution des éléments prélevés sur l'œuvre au sein d'un nouvelle syntaxe discursive, la substitution d'une saisie continue à celle, discontinue, par changements de places, de distances et de points de vue sur une sculpture par exemple - celle même que préconisait Benvenuto Cellini quand il parlait de la coexistence dans une ouvre en volume de quarante points de vue ${ }^{68}$, ou celle qu'un Auguste Choisy (suivi de Le Corbusier) théorisa sous le terme de "promenade " architecturale ${ }^{69}$-, l'inscription de l'approche de l'art sous la catégorie générale de montage. Quelques cinéastes et quelques historiens de l'art le crurent et en firent le pari : on voyait dans le film sur l'art en 1945 une nouvelle " avant-garde ", la mise en œuvre d'un langage nouveau. Mais les obstacles financiers et institutionnels, une certaine lourdeur en somme du médium, y mirent rapidement fin ${ }^{70}$ et, au final, la part la plus valorisée de la production des films sur l'art a, de plus en plus, été celle du "documentaire de création ", équivalent de l'essai en littérature, dont les exemples canoniques sont les courts métrages d'Alain Resnais et, à en croire Georges Sadoul ${ }^{71}$, plutôt

- 66 - Cf. " L'expérience consiste simplement à regarder, au travers d'une loupe généreuse, les petites têtes traitées par l'artiste [Watteau] dans le décor de scènes si enchanteresses. [...] examinezles avec le plus grand soin, agrandissez leurs traits par ce procédé mécanique : elles se transforment déjà » (D’Ors E., Du baroque, Paris, Gallimard, 1935 [rééd. 1968, p. 196]).

- 67 - L’essor de Google-Art est venu récemment le signifier en généralisant une formule qui ne passe pas par les démarches explicatives et pédagogiques que nombre de musées mettaient en place par ailleurs, mais se focalise avant tout sur l'image que propose le tableau, la fresque, la sculpture ou simplement l'objet en proposant de découvrir des détails « bien au-delà de ce qui est visible à l'œil nu " grâce aux sept milliards de pixels de définition par image (voir Herzberg N., "OPA à succès de Google sur les musées en ligne ", Le Monde, 15-16 avril 2012, p. 19).

- 68 - Cellini Benvenuto, "Traité de la sculpture " [1568], Euvres complètes, trad. Léopold Leclanché, tome II, Paris, Paulin, 1847, p. 398-399.

- 69 - Chorsy Auguste, Histoire de l'architecture, Paris, Gauthier-Villars, 1899, p. 412-420. Pour la reprise qu'en fit Le Corbusier, voir son ouvrage Vers une architecture, Paris, Crès, 1923, p. 153-154 (où il reprend un dessin de Choisy de l'Acropole sans le nommer) et Le Corbusier et Jeanneret Pierre, CEuvre complète de 1929-1934, Zurich, Les Éditions d'Architecture, 1995, p. 24-25.

- 70 - Il faudrait nuancer ce bilan en fonction des pays et de leurs régimes sociaux. Dans les pays socialistes où les questions de rentabilité n'intervenaient pas aussi directement sur la production, les films consacrés à l'artisanat, aux artistes nationaux ont continué d'alimenter une production documentaire soutenue par laquelle la plupart des cinéastes débutants passaient. On en connaît mal l'ampleur. Ainsi Andrzej Wajda signe-t-il plusieurs films de ce genre (tels La Poterie d'Ilza en 1951 et Je marche vers le soleil en 1955 sur le sculpteur Xavier Durikowski) qu'on découvre fortuitement au titre de bonus à l'occasion d'éditions DVD de ses films de fiction.

- 71 - Sadoul Georges, «L'art est-il anecdote ou réalité? Films sur l'art : Le Paradis perdu d'Emmer et Gras; De Renoir à Picasso de Paul Haesaerts; Gauguin de Resnais et Diehl; Guernica de Resnais, Hessens et Paul Éluard ", Les Lettres françaises, n 368, 21 juin 1951. 
Guernica (1950) - conjoignant la peinture de Picasso autour de sa fameuse toile de 1937, un poème de Paul Éluard, une musique de Guy Bernard, une interprétation dramatique de Maria Casarès - que son Gauguin (1950) ou son Van Gogh (1948). Quoique peu commentés, les cinq courts métrages de Jean Grémillon réalisés dans la même période, entre 1950 et 1958 - Les Charmes de l'existence (sur la peinture " pompier» des Salons du xix ${ }^{\mathrm{e}}$ siècle, avec Pierre Kast), Les Désastres de la guerre (avec Kast également), La Maison aux images, Haute-Lisse, et André Massonconstruisent un ensemble très cohérent sous-tendu par une réflexion cosmologique sur l'art comme geste, comme artisanat, comme rapport à la matière, aux éléments.

On a redistribué les fonctions de ce "genre » selon les besoins différenciés du didactisme scolaire ou muséal, de la vulgarisation des connaissances et de la reconduction d'une idéologie de la création héritée du $\mathrm{xIx}^{\mathrm{e}}$ siècle (cristallisée dans le biopic La Vie passionnée de Vincent Van Gogh). La documentation proprement dite, auxiliaire de l'exposition ou de la monographie, en dehors de quelques entreprises singulières (comme celle de Jean José Marchand et ses Archives du xx ${ }^{\mathrm{c}}$ siècle) ou de films documentaires exigeants (Painters Painting d'Emile De Antonio), s'est ramenée à deux figures : la saisie des gestes de l'artiste au travail (dans le temps court qui ne permet guère d'approcher réellement sa pratique) et, grâce à l'image électronique, l'analyse formelle par découpe et rajout à même la toile filmée d'éléments supposément successifs dans la genèse du tableau (dans un premier temps illustrée par Haesaerts et Storck puis Ragghianti et, de nos jours, avec la série Palettes d'Alain Jaubert).

Le « film sur l'art », s'il est devenu indispensable pour les institutions muséales, au sein des expositions (écrans) ou comme produits dérivés qu'on trouve en librairie (DVD), aura donc assez vite disparu des préoccupations des historiens de l'art, qui le retrouveront peut-être au sein des «visual studies» où se reconfigure leur discipline. Il n’a pas manqué, en revanche, d'intriguer les esthéticiens et les philosophes : le Matisse de François Campaux a ainsi généré un assez grand nombre de réflexions qui vont de Maurice Merleau-Ponty à André Bazin ${ }^{72}$. Indice d'un double déplacement, qui s'opère de la compréhension de l'œuvre et de ses liens

- 72 - L'étonnement que le film produisit, y compris auprès de Matisse lui-même, rencontrait en effet des problématiques de type philosophique : qu'est-ce que créer? Sur un plan plus terre à terre, voir Matisse dessiner retrouvait des apports du film d'enseignement à destination des apprentis : ainsi dans Le Fer forgé de Jean Benoît-Lévy (1922), Léon Moussinac voyait la démonstration que " certains tours de main exécutés rapidement ne sont vraiment saisissables que grâce au cinéma au ralenti. Rien ne peut plus échapper à l'élève des mouvements subtils de la main expérimentée qui exécute un travail difficile, utilise un outil pour un détail de technique particulièrement ardu, tandis que le maître explique. [...] Le cinéma a l'avantage, grâce au possible ralenti, de conserver au mouvement complet son unité et sa rigoureuse précision " (Moussinac L., "Le premier film d'enseignement. Le Fer forgé ", Cinémagazine, n 17, 28 avril 1922, p. 117). 
avec le contexte historique ou la carrière de l'artiste, vers, d'une part, la figure de cet artiste comme créateur voire démiurge ${ }^{73}$ - le Picasso de Clouzot, le Pollock de Namuth, plusieurs auto-mises en scène filmées de Dalí entre 1960 et $1975^{74}$ - et, d'autre part, vers le spectateur et son expérience de réception de tels films.

C'est alors de la part de cinéastes indépendants voire expérimentaux, que les dernières décennies ont vu venir des propositions de " films sur l'art " interrogeant les paramètres mêmes de ce type de représentation, interrogeant le cinéma (que peut-il en l'espèce?) comme cet objet (l'art, la réalité physique du tableau, l'acte ou le travail de la peinture ou de la sculpture) sans forcément se préoccuper de la représentativité du peintre choisi : dans Portrait du peintre dans son atelier (1985), Boris Lehman montre l'impossibilité de cette saisie; dans Cézanne (1989), Jean-Marie Straub et Danièle Huillet s'affrontent au filmage du " motif "du tableau et, dans Une visite au Louvre (2004), à l'objet-tableau lui-même (comment l'éclairer, le cadrer?), Johan van der Keuken associe le peintre Lucebert à son travail de documentariste et confronte sa peinture et ses dessins au monde réel ${ }^{75}$, dans El Sol del Membrillo (1992) Victor Erice enregistre la lutte du peintre et du temps, enfin Alexandre Sokourov explore l'univers de Hubert Robert à l'aide de lentilles déformantes (dans Hubert Robert, une vie heureuse, 1999) avant de s'attaquer au Louvre (Francofonia, 2015).

\section{Conclusion}

En 1959, Georges Sadoul écrivait : "Pour les historiens futurs, il se peut que les années 1950 aient été moins marquées par le cinémascope, le cinérama ou la stéréophonie que par l'avènement d'une "ciné-plastique" animant peinture, dessin, sculpture, gravure, etc. par tous les moyens que voulaient bien choisir l'artiste ou

- 73 - À l'occasion pour s'en moquer, comme des femmes-pinceaux d'Yves Klein et des compressions automobiles dans Mondo Cane (Paolo Cavara, Franco Prosperi et Gualtiero Jacopetti, 1962). - 74 - Le réalisateur Albert Serra a contribué à la redécouverte de ces " films sur l'art " daliniens où l'expérimental le dispute au portrait d'artiste : Chaos and Creation (Dalí et Philippe Halsman, 1960), Dali in New York (Jack Bond, 1965), Autoportrait mou de Salvador Dali (Jean-Christophe Averty, 1972) et Impressions de la Haute-Mongolie, hommage à Raymond Roussel (José MontesBaquer, 1975). En les programmant à la dOKUMENTA de Kassel 2012 et en marge de l'exposition sur Dalí du Centre Pompidou en 2013, il les décrivit comme "assez méconnus car ils échappent à toute approximation académique, ils sont un mélange d'imagination, de beauté et de vulgarité, typique de Dalí et à la fois contradictoire. Ils sont si fous que toute approximation archéologique ou fétichiste est également impossible * (Cité dans la brochure de la programmation cinéma/vidéo Albert Serra, 17 avril-12 mai 2013 du Centre Pompidou, "La carte blanche ", p. 20). - 75 - Sur cette photographie de tournage de Lucebert, temps et adieux (1994) où Van der Keuken double sa prise de vue par une prise en main de l'œuvre filmée, voir Albera F., "Sur trois photographies de Johan van der Keuken ", CiNéMAS, vol. 17, nº 1, automne 2006, p. 84-116. 
- 32 - F. Albera, L. Le Foresíer, V. Robert, K. Imesch ei M. Lüscher

le cinéaste ("l'image par l'image” n’étant qu’un procédé parmi plusieurs) peut-être plus parents des arts plastiques que du cinéma proprement dit, par la fusion de certains "films sur l'art" et du meilleur "cinéma d'animation"76. » Pressentiment d'une mutation technologique affectant moins la taille de l'image ou ses effets « hyperréalistes » (aujourd'hui rejoués sous les espèces du «3D») que la nature même du médium. Le spécialiste du cinéma d'animation, André Martin, ne disaitil pas, peu après ces propos de Sadoul, que la télévision allait libérer le cinéma de ses tâches de "représentation de la "vie" » et d' " enregistrement » des "feuilles qui bougent " et lui permettre de renouer avec... la chronophotographie, le dessin animé (qui a "de beaucoup précédé et préparé l'avènement de la photographie animée »), prenant l'exemple des films de deux plasticiens polonais, Jan Lenica et Walerian Borowczyk, qui s'étaient approprié le médium film ${ }^{77}$.

Ainsi en conclusion peut-on s'étonner qu'en regard des échanges nombreux, constamment évoqués, entre l'art et le cinéma, l'histoire de l'art ne se soit approchée de l'histoire du cinéma et du film lui-même qu'avec la plus grande retenue, voire qu'elle s'en soit totalement tenue à l'écart pendant une longue période - si l'on excepte le «moment Pollock ». Quand bien même, à titre individuel, d'importants historiens de l'art ont très tôt fait observer que l'histoire du cinéma était indissociable de celle de l'art, bien que de grands artistes $\mathrm{du} \mathrm{xx}^{\mathrm{e}}$ et $\mathrm{xxI}^{\mathrm{e}}$ siècle aient travaillé et travaillent avec le médium filmique, et qu'enfin bon nombre de représentants du cinéma et de la théorie cinématographique aient thématisé intensivement la relation diversifiée qu'ils entretiennent avec les arts plastiques et l'histoire de l'art ${ }^{78}$, cette dernière a mis longtemps à accepter sa cadette et son médium "inventé " à la fin du XIx ${ }^{\mathrm{e}}$ siècle $^{79}$. C'est ainsi qu'à l'exception de percées isolées l'intégration du film au "canon » des médias de l'histoire de l'art et de ses domaines de recherche ne s'est faite que lors de la réorientation vers les nouveaux médias les plus récents, ainsi qu'avec l'élargissement de la notion d'art survenue dans le cadre de la pensée dite " postmoderne ». Certes en 1934 déjà, aux États-Unis, Erwin Panofsky prononce une fameuse conférence sur les rapports de l'art et du cinéma à la demande d'Alfred Barr, directeur du Museum

- 76 - Sadoul G., "A Work in Progress ", Cahiers du cinéma, nº 91, janvier 1959, p. 50.

- 77 - Martin A., "Borowczyk et Lenica reviennent aux origines ", Cahiers du cinéma, n ${ }^{\circ}$ 96, juin 1959 , p. 35.

- 78 - Exemple récent, le recueil d'essais coordonné par Criqui J.-P. et MouëLlic G., "Le Cinéma surpris par les arts ", Les Cahiers du Musée national d'art moderne, été-automne 2010, n 112-113. - 79 - Certains aspects de cet effort d'évincement par l'histoire de l'art, dans l'espace germanophone, entre 1925 et 1950, sont reconstitués par Thomas Meder, ainsi que par certaines publications récentes d'histoire de l'art et interdisciplinaires (voir Meder Th., « Die Verdrängung des Films aus der deutschen Kunstwissenschaft 1925-1950 ", in РАECH J. [dir.], Film, Fernsehen, Video und die Künste. Strategien der Intermedialität, Stuttgart-Weimar, J. B. Metzler, 1994, p. 9-18). 
of Modern Art de New York, fondé quelques années plus tôt. Membre nouveau de l'Institute of Advanced Studies, Panofsky développa cette conférence et la publia à Princeton en 1936 sous le titre On Movies - et l'année suivante sous celui de Style and Medium in the Motion Pictures ${ }^{80}$ - mais cet important essai ne sera pleinement assimilé par l'histoire de l'art qu'au cours des années 1990. Le MoMA avait alors effectué une percée innovatrice dans cette direction, surprenante pour l'époque - 1935 -, avec l'ouverture d'un département cinéma dans ses murs : il incluait ainsi le jeune médium de masse dans le canon de l'histoire de l'art et dans le domaine d'activité d'un musée d'art moderne. Il faudra attendre les années 1980, avec le Centre Georges Pompidou à Paris puis le Getty Museum, pour que soit poursuivie la déclaration d'intention du MoMA des années 1930, selon laquelle il convenait de collectionner, cataloguer, analyser, exposer et présenter les films dans les musées, au même titre que les toiles, les sculptures et autres objets d'art ${ }^{81}$. Cette avancée du MoMA, qui souligne la compréhension artistique de cette institution nouvellement créée, était sous-tendue par une notion d'art étendue et peu orthodoxe, qui s'explique par la formation en histoire de l'art de son directeur fondateur, Alfred Barr, auprès du médiéviste Charles Rufus Morey au Department of Art and Archaeology de l'université de Princeton. Morey examinait l'art - comme l'Autrichien Aloïs Riegl - dans le contexte scientifique social, culturel et économique de sa création. Cette approche correspondait à ce qui était en jeu dans la pratique du mouvement Dada au début du xx ${ }^{c}$ siècle et anticipait en partie celle de ses successeurs - Néo-Dada, Pop Art, Nouveaux Réalistes, Fluxus et autres mouvements postmodernes de l'après-guerre ou, sur le plan de la théorie, avec l'avènement des Cultural and Visual Studies interdisciplinaires - intégration de l'art dans la vie quotidienne, dés-automatisation et assouplissement voire abandon complet de la séparation entre High et Low Art, art élevé, élitiste et art de masse. Ceci explique également pourquoi, dès les années 1930, le MoMA collectionna - outre des films - le design, les documents architecturaux, les objets quotidiens et la photographie et organisa des expositions pour familiariser le public avec

- 80 - Panofsky E., Style and Medium in the Motion Pictures, dans Three Essays on Style, Cambridge, Mass., The MIT Press, 1995 (traduction française : Trois essais sur le style, Paris, Le Promeneur, 1988). Pour un état des différentes éditions, voir Levin T. Y., "Iconology at the movies: Panofsky's Film Theory ", The Yale Journal of Criticism, vol. 9, $\mathrm{n}^{\circ}$ 1, 1996, p. 27-55.

- 81 - Voir aussi Wasson H., Museum Movies. The Museum of Modern Art and the Birth of Art Cinema, Berkeley, University of California Press, 2005. Il faut cependant relever le fait que dans de nombreux pays européens se mirent en place des archives cinématographiques proprement dites (cinémathèques soucieuses dans bien des cas de revendiquer le titre de "musée ") qui " dispensèrent " les musées d'art de se poser ce type de question au nom de la division du travail. À l'inverse il faut relever que la politique d'acquisition et de conservation du MoMA en matière de films procédait d'un choix selon des critères esthétiques qui se révélèrent par la suite limitatifs s'agissant d'un médium et média de masse. 
eux $^{82}$ - certaines de ces expositions, tel que l'International Style, faisant date dans l'histoire de l'art ou de l'architecture.

Ainsi la nouvelle orientation de l'histoire de l'art en direction du médium filmique a-t-elle été rendue possible par l'avènement de nouvelles lignes directrices théoriques et méthodologiques, reprises par l'histoire de l'art - telles les Visual and Cultural Studies et la Visual History qui, depuis les années 1990, ont abordé les films sous l'angle de l'étude de l'art et des images, sans pour autant laisser un grand impact sur le monde spécialisé. Ce qu'une telle approche peut représenter en termes de diversité et d'originalité est documenté par quelques études récentes au sujet de "l'image en mouvement » du point de vue de l'histoire de l'art, qui font bouger le discours scientifique et l'échange entre art et cinéma ${ }^{83}$. Elles ne se limitent pas non plus, à l'inverse de la plupart des travaux consacrés à la relation entre art et cinéma jusque-là, à l'analyse de biographies filmées d'artistes - fondées sur le modèle narratif des biographies cinématographiques d'artistes à la Vasari ${ }^{84}$ mais se servent de ce nouveau domaine d'étude pour mettre à l'épreuve une science critique des images.

Présenter de nouvelles méthodes d'analyse et questionner un genre cinématographique ou audiovisuel de manière interdisciplinaire - et donc aussi bien sous l'angle de l'histoire de l'art que de celle du film - telle est donc bien l'ambition de la présente publication.

- 82 - Bee H. S. et Elligotrt M. (dir.), Art in Our Time. A Chronicle of the Museum of Modern Art, New York, Museum of Modern Art, 2004.

- 83 - Voir, entre autres, Jones C. A., Machine in the Studio. Constructing the Postwar American Artist, Chicago/Londres, The University of Chicago Press, 1996; ou dans le domaine germanophone : Diers M., Fotografie Film Video. Beiträge zu einer kritischen Theorie des Bildes, Hambourg, EVA, 2006; Hensel Th., Krüger K. et Michalsky T. (dir.), Das bewegte Bild. Film und Kunst, Munich, Fink, 2006, qui comporte une bibliographie détaillée de travaux sur ce thème.

- 84 - Sur la construction formelle de ces biopics vasariens dont on a dit le succès historiographique fulgurant depuis les années 1980, voir notamment Felix J. (dir.), Genie und Leidenschaft. Künstlerleben im Film, St. Augustin, Gardez!, 2000, et Tayıor H. M., Rolle des Lebens. Die Filmbiographie als narratives System, Marburg, Schïren 2002. 


\section{Table des matières}

REMERCIEMENTS .7

F. Albera, L. Le Forestier, V. Robert, K. Imesch et M. Lüscher, Introduction: Approches d'un genre hybride, le film sur l'art.

\section{Aux origines du fillm sur l'art: didactisme ef propagande}

Valentine RoBerT,

L'histoire de l'art prise de vues.

Christel Taillibert,

Les films sur l'art produits en Italie au cours de la période fasciste. 55

Roxane HAMERY,

Les films sur l'art d'Afrique noire : entre colonialisme, ethnologie et histoire de l'art. France/Belgique (1945-1961) 


\section{2 \\ L'institutionnalisation du fillm sur l'art: entre histoire de l'art et cinéma}

Laurent Le Forestier,

Les films sur l'art en France après la Seconde Guerre mondiale:

allers-retours entre histoire de l'art et cinéma.

François Albera,

Pierre Francastel, un historien de l'art à la FIFA.

Frédéric Gimello-Mesplomb,

Les films sur l'art lauréats de la "Prime à la qualité " du CNC:

portrait d'une catégorie de bénéficaires de l'intervention culturelle publique (1954-1959).

\section{Le fillm sur l'art, création au second degré}

Fabienne Bonino,

Henri Storck : de la représentation cinématographique de la peinture, ou l'histoire de l'art renouvelée.

Céline Maes,

Paul Haesaerts et le film sur l'art: pour un "cinéma-critique"...

Chiara SAVETTIIERI,

Le "critofilm" de Carlo L. Ragghianti : une critique d'art visuelle.

Critique d'art et limites du langage....

Antonio Costa,

Venise : la peinture, la forme de la ville et le cinéma en forme d'essai.

Alain BoILlat,

Le discours sur l'art dans Ceux de chez nous de Sacha Guitry (1915-1952) :

la causerie d'un collectionneur. 
Benoît Turquety,

Travail du peintre, travail du cinéma:

Cézanne, Danièle Huillet et Jean-Marie Straub

\section{4 Déplacements du fillm sur l'art : actuallités, télévision et art vidéo}

Kornelia Imesch,

Les actualités mises à nu par ses Célibataires :

Jean Tinguely et la fin du "grand récit " suisse.

Mario LüsCHER,

Les figures du spectateur : l'art et son public dans les actualités filmées.

Yves Chevrefils Desbiolles,

Jean José Marchand et Les Archives du Xx ${ }^{\mathrm{e}}$ siècle.

Martin GouTTE,

Le culturel et l'actuel:

la place du "genre " dans deux journées spéciales d'Arte

Nicolas BrulHart,

Documenter l'art des néo-avant-gardes :

Gerry Schum, entre captation idéale et critique des dispositifs médiatiques.

\section{En guise de conclusion....}

Mario Lüscher et Valentine RoberT,

Histoire de l'art et films sur l'art:

entretien avec l'historienne de l'art et réalisatrice Judith Wechsler.

Filmographie

INDEX 
Achevé d'imprimer sur les presses du service reprographique de l'université Rennes 2 en janvier 2015.

Imprimé en France 


\section{Le film sur l'art \\ Entre histoire de l'art et documentaire de création}

Cet ouvrage dresse un état international des recherches menées sur ce « genre " cinématographique jusqu'ici peu étudié, hybride mais prolifique, au croisement de l'histoire de l'art et du cinéma documentaire : le film sur l'art.

Montrer l'artiste au travail, présenter la biographie d'un artiste disparu, sa vie intérieure, son œuvre, entrer dans la logique de celle-ci pour en déplier la thématique ou les narrations qu'elle contient, analyser sa construction formelle, aborder une période ou suivre des évolutions stylistiques : ce sont autant de démarches qui peuvent unir, selon des modalités diverses, un cinéaste et un artiste ou un cinéaste et un historien de l'art (Diehl, Schmidt, Cassou, Huyghe, Sweeney...) - quand l'historien de l'art ne se fait pas tout simplement cinéaste (Haesaerts, Ragghianti). C'est que, de Warburg à Panofsky, de Focillon à Elie Faure et à Malraux, de Longhi à Francastel et Damisch, nombreux sont les historiens de l'art qui se sont préoccupés de ce que le cinéma pouvait apporter à leur discipline, tant au plan de la simple documentation que du modèle de perception qu'il engage par le découpage - les détails, les changements d'échelles -, le montage, la mise en mouvement et la projection par transparence.

Quel bilan tirer de cette riche histoire du film sur l'art devenue quelque peu opaque (où sont ces films? comment les voir?) et que comprendre de l'histoire de ces productions qui, d'abord rattachées au documentaire, voire au cinéma pédagogique, ont ensuite trouvé une autonomie au sein du genre « film sur l'art »? Quels liens furent noués entre historiens de l'art, critiques d'art et réalisateurs? Quel apport à l'histoire de l'art cette production représente-t-elle? Comment l'histoire de l'art et ses applications dans différents médias circulent-elles dans ces films, et quel effet cet intérêt pour l'art a-t-il pu produire en retour sur le cinéma * comme art plastique »? Enfin, quelle est la situation présente de ce type d'approche? Ces questions sont ici collectivement posées, débattues, développées.

François ALBERA est professeur d'histoire et esthétique du cinéma à l'université de Lausanne, directeur de recherche au Fonds national de la recherche scientifique, membre de l'Association française de recherche sur l'histoire du cinéma et secrétaire de rédaction de 1895 revue d'histoire du cinéma.

Laurent LE FORESTIER est professeur en études cinématographiques à l'université Rennes 2, où il dirige le laboratoire de recherche en cinéma de l'équipe « Arts : pratiques et poétiques ". Il est membre du conseil d'administration de l'Association française de recherche sur l'histoire du cinéma et secrétaire d'édition de 1895 revue d'histoire du cinéma. Valentine RовеRт est chargée de cours en histoire et esthétique du cinéma à l'université de Lausanne, chercheuse invitée au GRAFICS de l'université de Montréal, doctorante FNS. Spécialiste de la relation peinture-cinéma, elle a contribué à de nombreux ouvrages, revues et expositions en montrant comment les films « réalisent » des tableaux vivants.
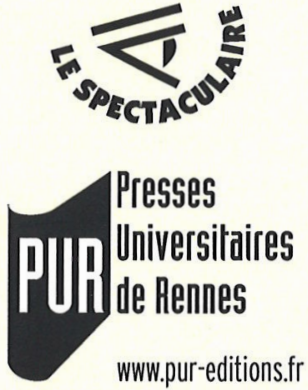

En couverture : Van der Keuken filmant et touchant une toile de Lucebert (photographie de tournage de Lucebert, temps et adieux, 1994).

ISBN 978-2-7535-3604-3

Ouvrage publié avec le soutien du programme spécifique de recherches, reconnu et financé par l'ANR : FILCREA

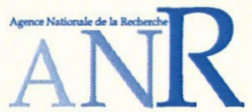

Prix : $20 €$

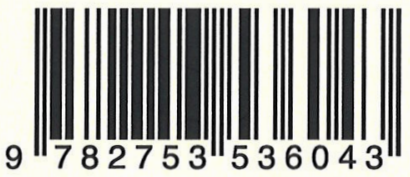

